\title{
Vibration analysis of an axially moving multiscale composite plate subjected to thermal loading
}

\author{
Krzysztof Marynowski, ${ }^{*}$ \\ Department of Dynamics, Lodz University of Technology, Poland
}

\begin{abstract}
This study investigated the effects of temperature on free vibrations and critical transport speeds of an axially moving multiscale composite plate. On the basis of the frequency-temperature equivalence principle, a linear mathematical model of the moving multiscale composite plate is derived in the complex frequency domain. Fractional standard rheological model of the plate material as the function of reduced frequency depended on the temperature is determined. In numerical investigations carbon nanotubes- and graphene-reinforced copper plate was taken into account.. To describe thermomechanical properties of the plate material, the investigation results obtained from the molecular dynamics studies and the experimental characteristic of beryllium copper in low temperatures presented in literature is taken into account.. The effects of temperature, transport speed, and internal damping on natural frequencies and critical transport speed are analyzed. The critical transport speeds of the graphene-reinforced multiscale composite are higher than both carbon nanotubes-reinforced composite as well as the comparable copper alloy.
\end{abstract}

\section{Introduction}

Axially moving composite objects can be found in many different technical applications. These include for example paper and textile webs during production and processing, conveyor belts, and flat objects moving at high speeds in space. Many factors influence the dynamic behavior of the moving object. The most important are: transport speed, tension, material properties and influence of external environment. Among the external factors affecting the dynamics, temperature plays an important role. Accurate identification of the temperature effect on the dynamics of the axially moving plant is important to properly control these devices. A literature review on dynamics of axially moving systems can be found, for example, in the study [1].

Studies in the field of dynamics of stationary composites are carried out for a long time. At the turn of the centuries composite materials began to appear particularly in the aerospace, maritime and space industries because they offer a number of advantageous mechanical properties., These properties include resistance to electrochemical corrosion, high strength and rigidity with less weight than conventional materials. The state of researches in the dynamics of laminated composite and sandwich plates in the paper [2] is presented.

In recent years, considerable research effort has been devoted to multiscale composites: carbon nanotubes (CNTs) and graphene-reinforced materials. Carbon nanotubes are very good reinforcement because of their unique mechanical properties and large surface area per unit volume. Experimental studies show that CNTs have Young's modulus greater than the graphite fibers, and strengths at least an order of magnitude higher than typical graphite fibers. Graphene attracted considerable interest because of its extraordinary mechanical, electronic and thermal properties. Experimental studies have shown that the single-layer graphene can present exceptional thermal conductivity which outperforms all other known materials.

For recent several years, graphene and CNTs have been used as reinforcement for polymeric composites because of their relative ease of processing. However, in recent times there has been increased interest in using matrices such as metals, semiconductors, and ceramics in addition to that of polymers. This study deals with metal matrix multiscale composites (MMMCs). Copper has been used as the metal matrix. MMMCs have less weight and higher strength in comparison to the conventional metals and alloys. MMMCs also have lower coefficient of friction and better resistance to wear and corrosion. Therefore these can be used in a variety of engineering applications such as automobile and aerospace industries. The state of research on mechanical and tribological properties of MMMCs in the paper [3] is presented.

The objective of this study is vibration analysis of the axially moving multiscale composite plate taking into account thermal effects. To describe thermomechanical properties of the plate material, properties of multiscale reinforced stationary composites presented in literature are taken into account. Fractional standard rheological model of the plate material as the function of reduced frequency depended on the temperature is taken into considerations. The effects of temperature, transport speed, and internal damping on natural frequencies and critical transport speed are analyzed.

\footnotetext{
Corresponding author: krzysztof.marynowski@p.lodz.pl
} 


\section{Factional standard rheological model}

The basis dependence of linear viscoelasticity, that the stress function is linearly dependent on the past history of the strain function, is expressed by Boltzmann's superposition principle in the form

$$
\sigma(t)=\int_{0}^{t} r(t-\tau) \frac{\partial \varepsilon(\tau)}{\partial \tau} d \tau
$$

where: $\sigma(\mathrm{t})$ - stress function, $\varepsilon(\mathrm{t})-$ strain function, $\mathrm{r}(\mathrm{t})-$ relaxation function. From the mathematical point of view Eq. (1) is a convolution of functions, thus using Laplace transformation one can find equivalent of Eq. (1) in the complex frequency domain

$$
\sigma(\omega)=R(\omega) \varepsilon(\omega),
$$

where: $R(\omega)$ - transform of relaxation function in the frequency domain. On the base of Eq. (2) one can solve viscoelasticity problems as elasticity problems using a complex modules of relaxation function which depends on frequency. Eq. (2) defines the elastic - viscoelastic equivalence principle.

Most often in studies of dynamics of viscoelastic systems to determine the complex moduli the fractional model is used [4]. To model the behavior of the moving plate under thermal loading the fractional standard rheological model was used in this paper, whereas the hydroelastic behavior was considered as elastic

The constitutive equation of the fractional standard rheological model

$$
\frac{\gamma}{E_{2}} \frac{d^{\beta} \sigma}{d t^{\beta}}+\sigma=\frac{\gamma\left(E_{1}+E_{2}\right)}{E_{2}} \frac{d^{\beta} \varepsilon}{d t^{\beta}}+E_{1} \varepsilon
$$

where: $0<\beta<1$. Taking into account the relaxation time constants

$$
\tau_{1}=\frac{\gamma}{E_{1}} ; \quad \tau_{2}=\frac{\gamma}{E_{2}}: \quad \tau_{0}=\tau_{1}+\tau_{2},
$$

the constitutive equation has the following form:

$$
\tau_{2} \frac{d^{\beta} \sigma}{d t^{\beta}}+\sigma=E_{1}\left(\tau_{0} \frac{d^{\beta} \varepsilon}{d t^{\beta}}+\varepsilon\right)
$$

In the free vibration problem the stress and strain functions can be expressed as

$$
\sigma=\sigma_{0} \exp (i \omega t) ; \quad \varepsilon=\varepsilon_{0} \exp (i \omega t) .
$$

In accordance with the frequency-temperature equivalence principle, the frequency $\omega$ can be replaced by the reduced frequency $\omega \alpha(T)$ depended on the temperature. Substituting Eqs (6) into the constitutive equation (5) one receives the Young's modulus in the frequency domain

$$
E(\omega, T)=\frac{\sigma_{0}}{\varepsilon_{0}}=E_{1} \frac{1+\tau_{0}(i \omega \alpha(T))^{\beta}}{1+\tau_{2}(i \omega \alpha(T))^{\beta}} .
$$

On the base of eq. (7) Fig.1 shows the shift of the storage Young modulus for different four temperatures in the frequency domain. The highest curve corresponds to the lowest temperature $T_{1}$ and vice versa the lowest curve corresponds to the highest temperature $T_{4}$.

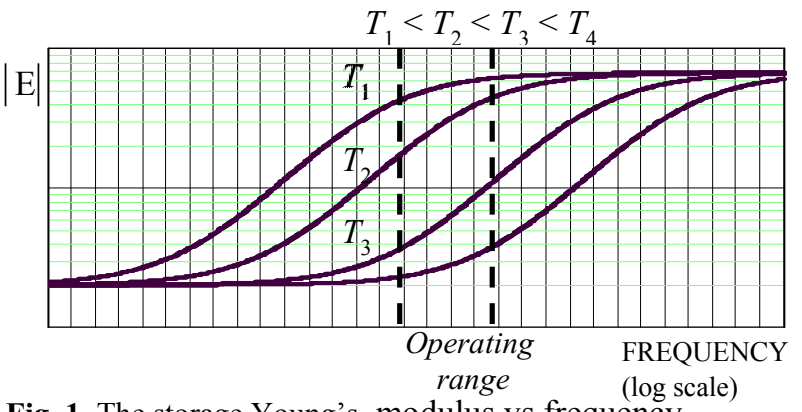

Fig. 1. The storage Young's. modulus vs frequency

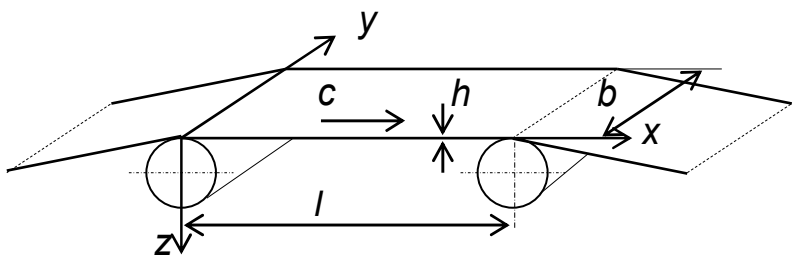

Fig. 2. Axially moving plate

\section{Mathematical model of the plate}

A thin multiscale composite plate of the length $l$, the width $b$ and the thickness $h$ is considered. The plate moves along the longitudinal direction $x$ at the constant velocity $c$. The co-ordinate system and the geometry are shown in Fig. 2.

The partial differential equation resulting from the Hamilton's principle for a transverse motion of the twodimensional axially moving plate was derived in the book [5] and has the following form:

$$
\begin{aligned}
& \rho h\left(\frac{\partial^{2} \bar{w}}{\partial t^{2}}+2 c \frac{\partial^{2} \bar{w}}{\partial x \partial t}+c^{2} \frac{\partial^{2} \bar{w}}{\partial x^{2}}\right)-\frac{\partial\left(N_{x} \bar{w}_{, x}\right)}{\partial x}-\frac{\partial\left(N_{y} \bar{w}_{, y}\right)}{\partial y}- \\
& -\frac{\partial\left(N_{x y} \bar{w}_{x}\right)}{\partial y}-\frac{\partial\left(N_{x y} \bar{w}_{, y}\right)}{\partial x}-\frac{\partial^{2} M_{x}}{\partial x^{2}}-2 \frac{\partial^{2} M_{x y}}{\partial x \partial y}-\frac{\partial^{2} M_{y}}{\partial y^{2}}=0
\end{aligned}
$$

where: $\rho$ - mass density; $h$ - thickness; wtransverse displacement of the plate; $M_{\mathrm{x}}, M_{\mathrm{y}}, M_{\mathrm{xy}}-$ bending moments per unit length; $N_{\mathrm{x}}, N_{\mathrm{y}}, N_{\mathrm{xy}}$-in-plane forces per unit length.

The equilibrium state of the moving plate is defined as the non-trivial equilibrium position when timedependent forces do not interact on the plate and the form of deflection is dependent only on the transport speed. The equation of equilibrium states of the axially moving plate with a constant speed which is tensioned with the constant longitudinal load $N_{\mathrm{x} 0}$ can be defined on the basis of governing Eq. (8) in the form:

$\rho h c^{2} \frac{\partial^{2} w}{\partial x^{2}}-N_{x 0} \frac{\partial^{2} w}{\partial x^{2}}-\frac{\partial^{2} M_{x}}{\partial x^{2}}-2 \frac{\partial^{2} M_{x y}}{\partial x \partial y}-\frac{\partial^{2} M_{y}}{\partial y^{2}}=0$

Bending moments can be expressed as:

$$
\begin{aligned}
& M_{x}=\int_{-h / 2}^{h / 2} \sigma_{x} z d z, \quad M_{y}=\int_{-h / 2}^{h / 2} \sigma_{y} z d z, \\
& M_{x y}=\int_{-h / 2}^{h / 2} \sigma_{x y} z d z .
\end{aligned}
$$

Taking into account the thin-plate theory, Eqs (10) have the forms: 


$$
\begin{aligned}
& M_{x}=-D(\omega, T)\left(\frac{\partial^{2} w}{\partial x^{2}}+\eta v(\omega, T) \frac{\partial^{2} w}{\partial y^{2}}\right), \\
& M_{y}=-\eta D(\omega, T)\left(\frac{\partial^{2} w}{\partial y^{2}}+v(\omega, T) \frac{\partial^{2} w}{\partial x^{2}}\right), \\
& M_{x y}=-D_{1}(\omega, T) \frac{\partial^{2} w}{\partial x \partial y},
\end{aligned}
$$

where: $\eta$ - orthotropy coefficient,

$$
\begin{aligned}
& D(\omega, T)=\frac{h^{3}}{12} \frac{E(\omega, T)}{\left(1-\eta \nu(\omega, T)^{2}\right)}, \\
& D_{1}(\omega, T)=\frac{G(\omega, T) h^{3}}{6} .
\end{aligned}
$$

In the free vibration problem, the transverse displacement can be defined as:

$$
w(x, y, t)=w(x, y, \omega) \exp (i \omega t)
$$

Substituting Eqs (11) into governing Eq. (9), one receives a differential equation of equilibrium states of the moving plate in the frequency domain:

$$
\begin{aligned}
& \rho h c^{2} \frac{\partial^{2} w}{\partial x^{2}}-N_{x 0} \frac{\partial^{2} w}{\partial x^{2}}+D(\omega, T) \times \\
& {\left[\frac{\partial^{4} w}{\partial x^{4}}+2\left(\eta v(\omega, T)+\frac{D_{1}(\omega, T)}{D(\omega, T)}\right) \frac{\partial^{4} w}{\partial x^{2} \partial y^{2}}+\eta \frac{\partial^{4} w}{\partial y^{4}}\right]=0 .}
\end{aligned}
$$

Eq. (14) can be transposed to a dimensionless form using the following terms:

$$
\begin{aligned}
& z=\frac{w}{h} ; \xi=\frac{x}{l} ; \varphi=\frac{y}{l} ; r=\frac{b}{l} ; c_{w}=\sqrt{\frac{N_{x 0}}{h \rho}} ; s=\frac{c}{c_{w}}=c \sqrt{\frac{h \rho}{N_{x 0}}} ; \\
& \tau=\frac{t c_{w}}{l} ; \omega=\frac{\omega l}{c_{w}} ; \Delta(\omega, T)=\frac{D(\omega, T)}{N_{x 0} l^{2}} ; \Delta_{1}(\omega, T)=\frac{D_{1}(\omega, T)}{N_{x 0} l^{2}} .
\end{aligned}
$$

The dimensionless equation of the equilibrium state in the frequency domain has the form:

$$
\begin{aligned}
& \left(s^{2}-1\right) \frac{\partial^{2} z}{\partial \xi^{2}}+\Delta(\omega, T) \times \\
& {\left[\frac{\partial^{4} z}{\partial \xi^{4}}+2\left(\eta \nu(\omega, T)+\frac{\Delta_{1}(\omega, T)}{\Delta(\omega, T)}\right) \frac{\partial^{4} z}{\partial \xi^{2} \partial \varphi^{2}}+\eta \frac{\partial^{4} z}{\partial \varphi^{4}}\right]=0}
\end{aligned}
$$

The dimensionless boundary conditions referring to simple supports at transverse ends and longitudinal free ends are as follows:

$$
\begin{aligned}
& \left.z\right|_{\substack{\xi=0 \\
\xi=1}}=0 ;-\left.\Delta(\omega, T)\left(\frac{\partial^{2} z}{\partial \xi^{2}}+v(\omega, T) \frac{\partial^{2} z}{\partial \varphi^{2}}\right)\right|_{\substack{\xi=0 \\
\xi=1}}=0, \\
& -\left.\Delta(\omega, T)\left(\frac{\partial^{2} z}{\partial \varphi^{2}}+v(\omega, T) \frac{\partial^{2} z}{\partial \xi^{2}}\right)\right|_{\substack{\varphi=0 \\
\varphi=r}}=0 ; . \\
& -\left.\Delta(\omega, T)\left(\frac{\partial^{3} z}{\partial \varphi^{3}}+(2-v(\omega, T)) \frac{\partial^{3} z}{\partial \xi^{2} \partial \varphi}\right)\right|_{\substack{\varphi=0 \\
\varphi=r}}=0 .
\end{aligned}
$$

Comparing Eq.(14) with the equation of motion for a stationary plate subjected to in-plane tension along the longitudinal direction, a necessary condition for the existence of non-trivial equilibrium positions of the plate can be obtained in the following form:

$$
s^{2}-1 \geq 0 \quad \text { or } \quad c \geq \sqrt{\frac{N_{x 0}}{\rho h}} .
$$

Assuming the notation:

$$
\psi(\omega, T)=\frac{s^{2}-1}{\Delta(\omega, T)},
$$

the equilibrium state equation has the form:

$$
\begin{aligned}
& \psi(\omega, T) \frac{\partial^{2} z}{\partial \xi^{2}}+\frac{\partial^{4} z}{\partial \xi^{4}}+ \\
& 2\left(\eta \nu(\omega, T)+\frac{\Delta_{1}(\omega, T)}{\Delta(\omega, T)}\right) \frac{\partial^{4} z}{\partial \xi^{2} \partial \varphi^{2}}+\eta \frac{\partial^{4} z}{\partial \varphi^{4}}=0 .
\end{aligned}
$$

The solution to Eq. (20) enables us to determine non-trivial equilibrium positions of the plate $z_{\mathrm{nk}}(n, k=1$, $2, \ldots)$ complying with multiple values $\psi(\omega, T)_{\mathrm{nk}}$. The solution to Eq.(20) is forecast in the form:

$$
z_{n k}(\xi, \varphi, \omega)=A_{k} \exp (k(\omega) \varphi) \sin (n \pi \xi) .
$$

Substituting Eq. (21) into equation (20), the fourthorder characteristic equation is obtained:

$$
k^{4}-2 n^{2} \pi^{2}\left(v(\omega, T)+\frac{\Delta_{1}(\omega, T)}{\eta \Delta(\omega, T)}\right) k^{2}+\frac{n^{4} \pi^{4}}{\eta}-\frac{n^{2} \pi^{2}}{\eta} \psi(\omega, T)=0 .
$$

Having introduced the notations:

$$
\Delta_{2}(\omega, T)=v(\omega, T)+\frac{\Delta_{1}(\omega, T)}{\eta \Delta(\omega, T)} ; \Delta_{3}(\omega, T)=\Delta_{2}^{2}(\omega, T)-\frac{1}{\eta},
$$

the solutions of characteristic Eq. (23) have the forms:

$$
\begin{aligned}
& \alpha_{1 n}=\sqrt{\Delta_{2}(n \pi)^{2}+n \pi \sqrt{\Delta_{3}(n \pi)^{2}+\psi / \eta}} ; \\
& \alpha_{2 n}=\sqrt{\Delta_{2}(n \pi)^{2}-n \pi \sqrt{\Delta_{3}(n \pi)^{2}+\psi / \eta}} ; \\
& \alpha_{3 n}=\sqrt{-\Delta_{2}(n \pi)^{2}+n \pi \sqrt{\Delta_{3}(n \pi)^{2}+\psi / \eta}}
\end{aligned}
$$

Solutions (24) comply with three sets of non-trivial equilibrium positions:

- for $0 \leq \psi<(n \pi)^{2}$ :

$$
\begin{aligned}
z_{n 1} & =\left[C_{11} \sinh \left(\alpha_{1} \varphi\right)+C_{12} \cosh \left(\alpha_{1} \varphi\right)+\right. \\
& \left.C_{13} \sinh \left(\alpha_{2} \varphi\right)+C_{14} \cosh \left(\alpha_{2} \varphi\right)\right] \sin (n \pi \xi),
\end{aligned}
$$

- for $\psi>(n \pi)^{2}$ :

$$
\begin{aligned}
z_{n 2}= & {\left[C_{21} \sinh \left(\alpha_{1} \varphi\right)+C_{22} \cosh \left(\alpha_{1} \varphi\right)+\right.} \\
& \left.C_{23} \sin \left(\alpha_{3} \varphi\right)+C_{24} \cos \left(\alpha_{3} \varphi\right)\right] \sin (n \pi \xi),
\end{aligned}
$$

- for $\psi=(n \pi)^{2}$ :

$$
\begin{aligned}
z_{n 3}= & {\left[C_{31} \sinh \left(\alpha_{1} \varphi\right)+C_{32} \cosh \left(\alpha_{1} \varphi\right)+\right.} \\
& \left.C_{33}+C_{34} \varphi\right] \sin (n \pi \xi),
\end{aligned}
$$

where: $C_{11}, C_{12}, \ldots . . C_{34}$ - constants, which are determined from boundary conditions (17). Fig. 3 shows the plots of $z_{11}, z_{12}, z_{21}$ and $z_{22}$ equilibrium states of the moving composite plate. To determine coefficients $\psi_{\mathrm{n}}$ which describe non-trivial equilibrium positions, Eqs (25) - (27) are substituted into boundary conditions (17). The critical transport speed is the lowest transport speed at which the non-trivial equilibrium positions exist. 

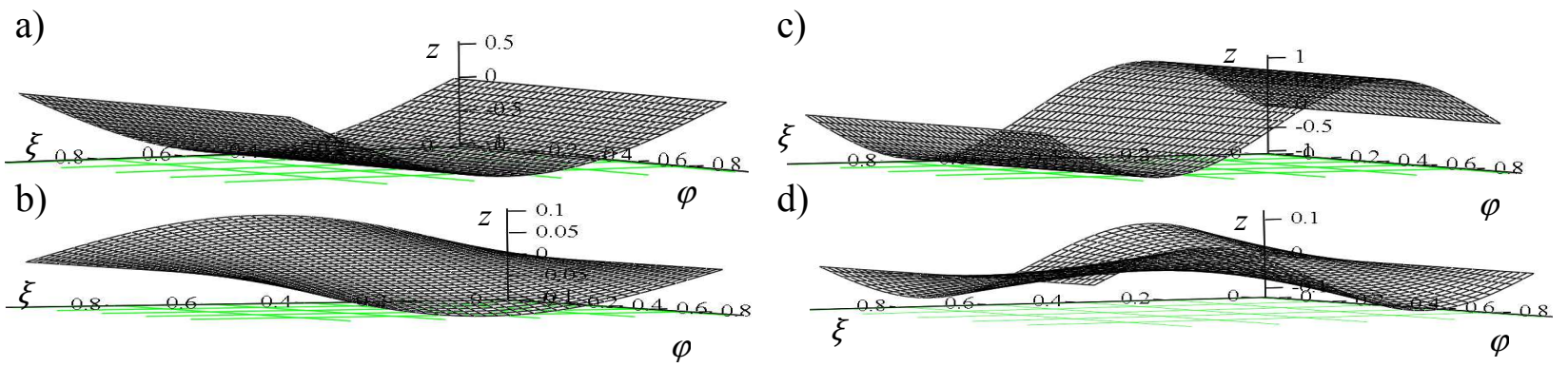

Fig. 3. Deflection forms at equilibrium states: $z_{11}$ (a), $z_{12}$, (b), $z_{21}$ (c), $z_{22}$ (d).

\section{Investigation results}

To describe thermomechanical properties of the plate material, the investigation results obtained from the molecular dynamics studies presented in literature are taken into account. Thermomechanical properties of the multiscale materials presented in the study [6] are shown in Tab.1 and 2. The single-layer graphene sheets (SLGS) and carbon nanotubes were appled as nanofillers in copper matrix in this studies. The Young's and Kirchhoff's moduli are summarized in Table 1 along with the analogical results of pure $\mathrm{Cu}$. Table 2 lists the Young's modulus in the axial direction of these multiscale composites at different temperatures along with the results of pure $\mathrm{Cu}$ for comparison.

Table 1. Comparison of mech. properties of $\mathrm{Cu}$ based nanocomposite reinforced with $9 \%$ vol. fraction of nanofiller [6]

\begin{tabular}{|l|c|c|c|c|}
\hline & $\begin{array}{l}\mathrm{E}_{11} \\
{[\mathrm{GPa}]}\end{array}$ & $\begin{array}{l}\mathrm{E}_{22} \\
{[\mathrm{GPa}]}\end{array}$ & $\begin{array}{l}\mathrm{G}_{23} \\
{[\mathrm{GPa}]}\end{array}$ & $\begin{array}{l}\mathrm{G}_{12} \\
{[\mathrm{GPa}]}\end{array}$ \\
\hline Pure $\mathrm{Cu}$ & 56 & 56 & 22 & 22 \\
\hline SLGS-Cu & 164.8 & 93.5 & 26.3 & 23.7 \\
\hline CNT-Cu & 140.7 & 67.9 & 24.2 & 22.6 \\
\hline
\end{tabular}

Table 2. Young's modulus ( $\left.\mathrm{E}_{11}[\mathrm{GPa}]\right)$ of $\mathrm{Cu}$-based nanocomposite at different temperatures [6]

\begin{tabular}{|l|c|c|c|}
\hline & \multicolumn{3}{|c|}{ Temperature ["K] } \\
\hline & $1 \mathrm{~K}$ & $300 \mathrm{~K}$ & $500 \mathrm{~K}$ \\
\hline Pure $\mathrm{Cu}$ & 86 & 56 & 45 \\
\hline SLGS-Cu & 175.4 & 164.8 & 156.4 \\
\hline CNT-Cu & 150.4 & 140.7 & 131.2 \\
\hline
\end{tabular}

Numerical investigations of free vibrations were carried out the axially moving multiscale copper plate shown in Fig.2. The plate is tensioned with the constant longitudinal load $N_{\mathrm{x} 0}$. Parameters of the moving plate are presented in Table 3.

Table 3. Parameters of the plate

\begin{tabular}{|c|c|c|c|}
\hline Parameter & Units & Notation & Value \\
\hline thickness & $\mathrm{mm}$ & $h$ & 0.8 \\
\hline length & $\mathrm{m}$ & $l$ & 1.0 \\
\hline width & $\mathrm{m}$ & $b$ & 0.1 \\
\hline axial load & $\mathrm{N} / \mathrm{m}$ & $N_{\mathrm{x} 0}$ & 100 \\
\hline Cu mass density & $\mathrm{kg} / \mathrm{m}^{3}$ & $\rho$ & 8930 \\
\hline wave velocity & $\mathrm{m} / \mathrm{s}$ & $c_{\mathrm{w}}$ & 3.74 \\
\hline
\end{tabular}

\subsection{Identification of the fractional model}

To identify the parameters of the fractional rheological model of the plate material, analysis of dynamics of the beam model of this structure was carried out [5]. Fig.4 shows the plot of the lowest natural frequencies of the beam model of the pure copper plate vs transport speed in the ambient temperature. On the base of these results the first natural frequency of the stationary beam model $\omega_{10}$ was treated as the frequency coordinate of the operating point on the storage Young's modulus plot in the ambient temperature. The low- and high-frequency asymptotes of this plot constitute the values of the Young's moduli from the molecular dynamic study for $500 \mathrm{~K}$ and $1 \mathrm{~K}$, respectively. The frequency characteristic in the ambient temperature with different slop was carried out by the operating point. Fig. 5 shows the characteristic of the pure copper plate for the $\operatorname{slop} \beta=0.03$.

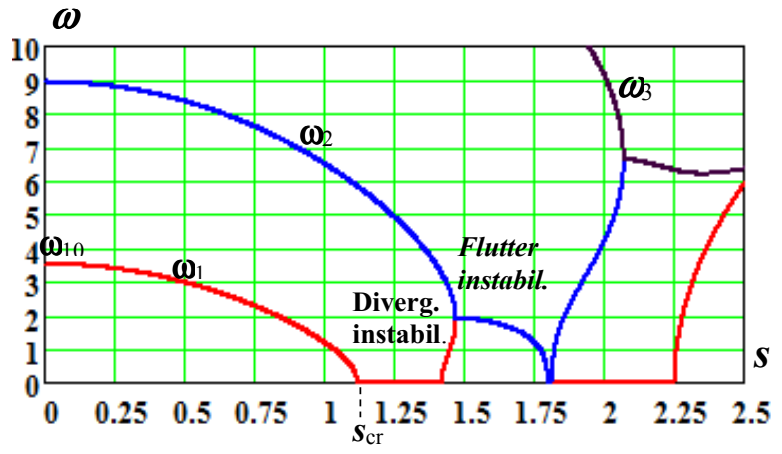

Fig. 4. The lowest natural frequencies of the beam model of the pure copper plate vs transport speed

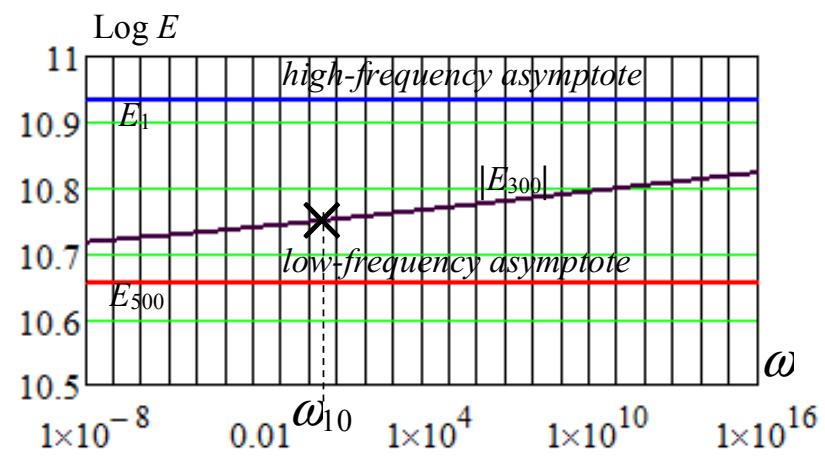

Fig. 5. The storage Young's modulus vs frequency (pure $\mathrm{Cu}$, $T=300 \mathrm{~K}, \beta=0.03$ ) 
The temperature dependent the reduced frequency factor in the fractional model of the plate material was determined by the Willims-Ferre-Lendel method using the formula

$$
\log [\alpha(T)]=\frac{c_{1}\left(T-T_{0}\right)}{c_{2}+T-T_{0}}
$$

where: $\mathrm{c}_{1}$ and $\mathrm{c}_{2}$ - experimental constants, $T$ - measuring temperature, $T_{0}$ - reference temperature.

However, in the literature very limited information on the temperature characteristics of the multiscale composites can be found. To determine the values of the experimental constants $C_{1}$ and $C_{2}$ in equations (28) the experimental characteristic of beryllium copper from the references [7] was used (Fig.6.).

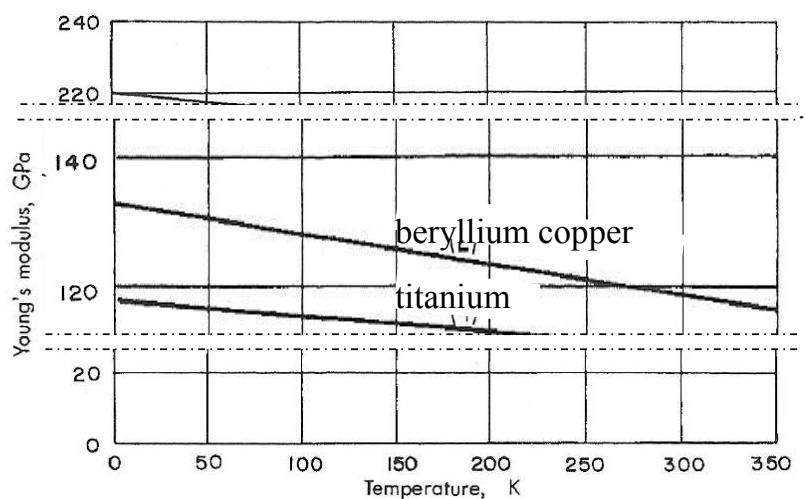

Fig. 6. The plot of the Young's modulus vs temperature for beryllium copper [7]

To determine the values of experimental constants for multiscale copper composites the same experimental characteristic of the copper alloy in low temperatures was taken into consideration.

\subsection{Results of numerical investigations}

Fig.7 shows the graphs of the decimal logarithm of the determinant of the matrix $\mathrm{Q}$ received from boundary conditions versus the frequency value for the three lowest modes.

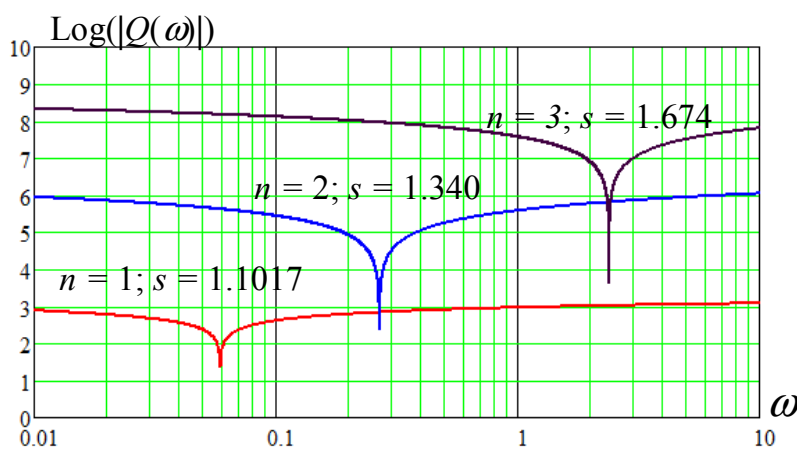

Fig.7. Non-trivial equilibrium positions (pure $\mathrm{Cu}$, material properties from molecular dynamic study, $T=350 \mathrm{~K}, \tau=$ $0.1487, \tau_{0}=0.2842, \beta=0.03$ )

The local minima on the curves in Fig.7 determine the natural frequencies of vibrations. The critical transport speed of the plate corresponds to the speed at which the lowest natural frequency vanishes. In numerical investigations the transport speed at which the lowest natural frequency equals 0.01 has been treated as the critical speed. Fig. 8 illustrates the procedure of determining the critical transport speed of the copper plate.

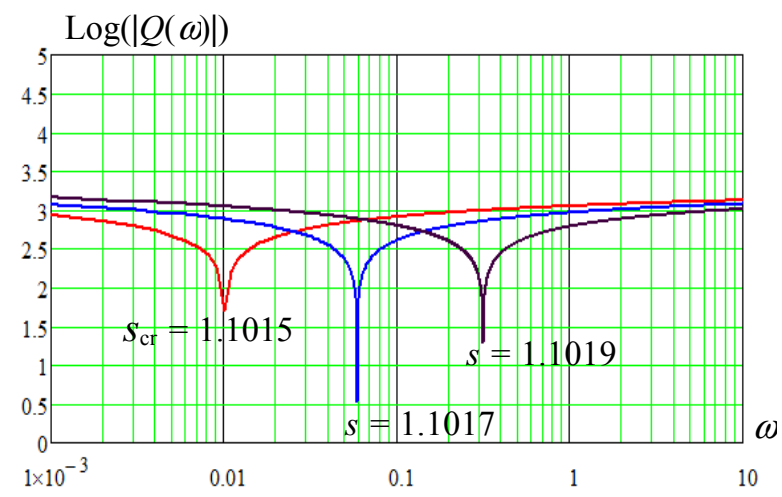

Fig. 8. Determining the critical transport speed of the moving copper plate (material properties from molecular dynamic study, $T=350 \mathrm{~K}, n=1, \tau=0.1487, \tau_{0}=0.2842, \beta=0.03$ )

Results of numerical investigation in Fig.9 show effect of temperature on the lowest non-trivial equilibrium positions of three successive modes for the pure copper plate. The lowest solid curve presents the plot of the critical transport speed versus temperature.

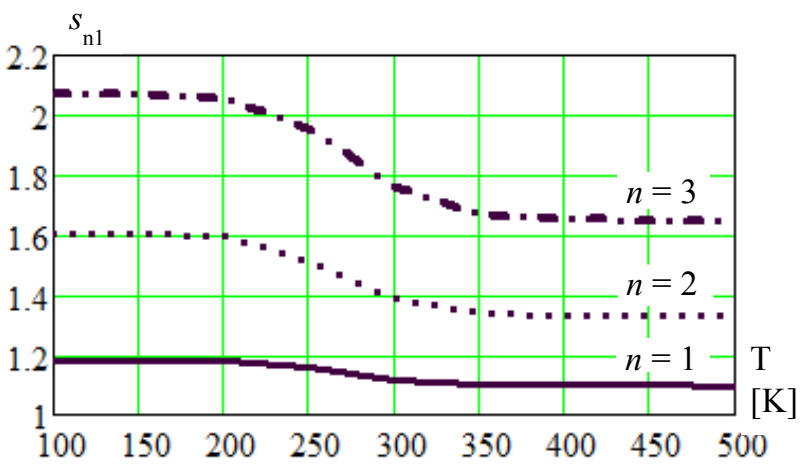

Fig. 9. Effect of temperature on the lowest non-trivial equilibrium position (pure $\mathrm{Cu}$ - material properties from molecular dynamic study, $\beta=0.03$ )

The plot of the critical transport speed for the pure copper plate is also visible in Fig.10. This is comparison of investigation results of critical speeds for both multiscale plates, beryllium copper and copper plates.

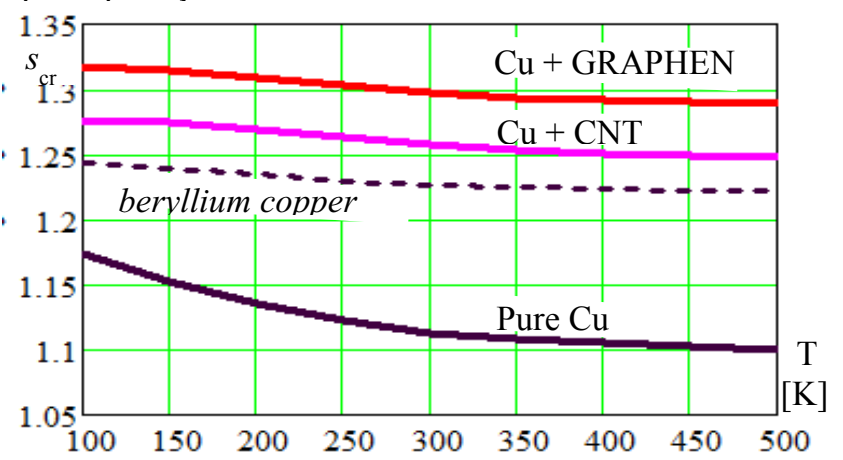

Fig.10. Effect of temperature on the critical transport speed (material properties from molecular dynamic study solid line, and experiment - dashed line, $\beta=0.01$ ) 
The plots in Fig. 10 show that critical transport speeds of all investigated plates decrease with increasing temperature. In the considered temperature range, the critical speeds of the graphene-reinforced composite are higher than the others. Figures 11, 12 and 13 show effect of temperature and the slope of the frequency characteristics on the critical speeds.

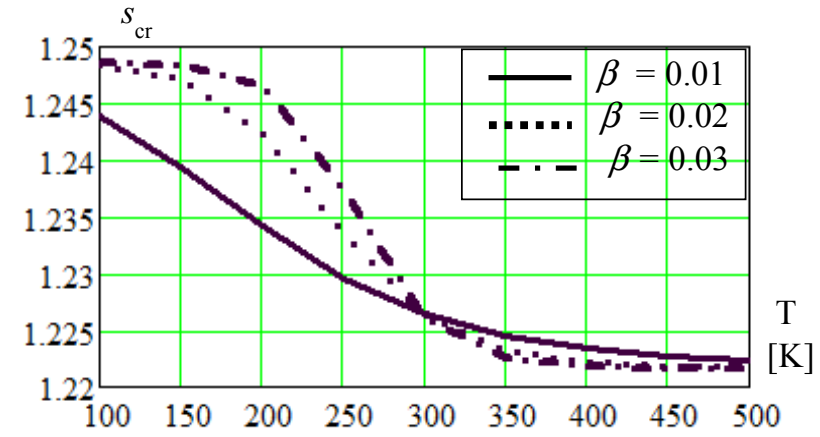

Fig.11. Effect of temperature and the rheological parameter $\beta$ on the critical transport speed for beryllium copper

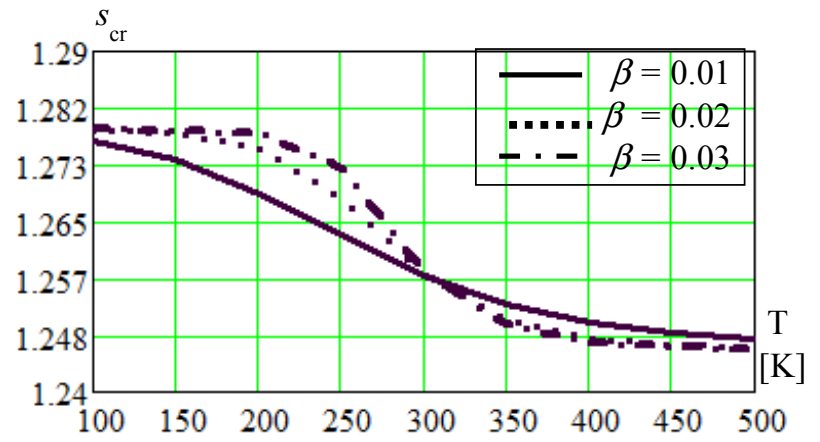

Fig.12. Effect of temperature and the rheological parameter $\beta$ on the critical transport speed for the $\mathrm{CNT} / \mathrm{Cu}$ composite

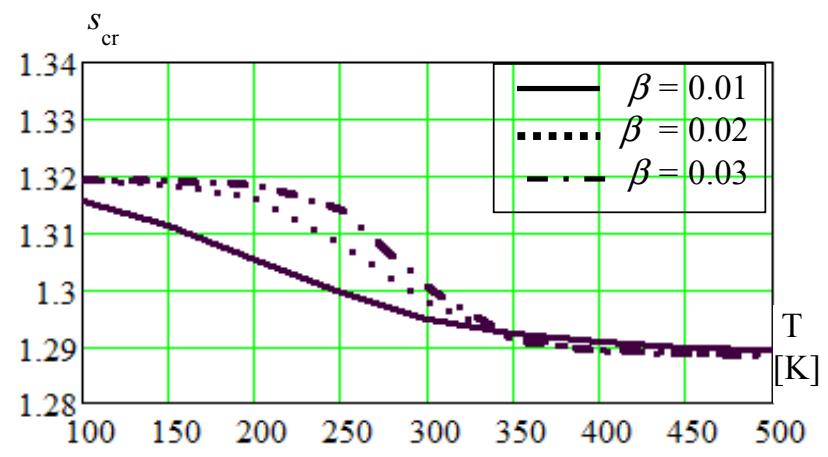

Fig.13. Effect of temperature and the rheological parameter $\beta$ on the critical transport speed for the graphene $/ \mathrm{Cu}$ composite

Figures 11, 12 and 13 show increasing the slope of the frequency characteristics results in an increase the value of the critical speeds at lower temperatures and reducing this value at the higher temperatures.

\section{Conclusions}

Although recently big research effort has been devoted to multiscale composites, there is still a need to know the dynamics of these structures. Dynamics of the axially moving multiscale composite plate subjected to thermal load is analyzed in this study. To describe thermomechanical properties of the plate material, properties of multiscale stationary composites presented in the literature are taken into account. Fractional standard rheological model of the plate material as the function of reduced frequency depended on the temperature is determined. On the base of the Hamilton's principle for the transverse motion the equation of equilibrium states of the plate in the frequency domain is derived.

Research plants were CNT-reinforced and graphenereinforced metal composites with copper matrix. In the literature very limited information on the temperature characteristics of these composites can be found. Therefore, to determine the temperature-depended reduced frequency in the fractional rheological model, the experimental characteristic of beryllium copper in low temperatures was used.

The free vibrations of multiscale composite plates are analyzed. Critical transport speeds of both multiscale composites decrease with increasing temperature. In the examined temperature range, the critical transport speeds of the graphene-reinforced composite are higher than both the CNT-reinforced composite as well as the comparable copper alloy. Increasing the slope of the frequency characteristics of all composites results in an increase of the critical transport speeds in low temperatures and a lower reducing the critical transport speed in the high temperature range.

\section{References}

1. K. Marynowski, T. Kapitaniak. Int. J. Mech. Sc. 81, 26 (2014)

2. A.S. Sayyad, Y.M. Ghugal, Compos. Struct. 129, 177 (2015)

3. A.D. Moghadam, E. Omrani, P.L Menezes, P.K Rohatgi. Comp Part B, 77, 402, (2015)

4. T .Pritz. J, Sound and Vibr. 195(1), 103 (1996)

5. K. Marynowski..Dynamics of the axially moving orthotropic web. (Springer-Verlag: 2008)

6. S, Sharma, P. Kumar, R. Chandrai. J Comp. Mater, DOI: IO.1177/0021998316682363 (2016),

7. S. Van Sciver, J. Pfotenhauer USPAS Cryogenics Short Course, Boston, MA 6/14/2010 - 6/18/2010 http://uspas.fnal.gov/materials/10MIT/Lecture_1.2. pdf 